\title{
UNIFORMLY CONVERGENT HIGHER-ORDER FINITE DIFFERENCE SCHEME FOR SINGULARLY PERTURBED PARABOLIC PROBLEMS WITH NON-SMOOTH DATA
}

\author{
Tesfaye Aga Bullo ${ }^{1}$, Guy Aymard Degla ${ }^{2}$, Gemechis File Duressa ${ }^{1}$ \\ ${ }^{1}$ Department of Mathematics, Jimma University, Jimma, Ethiopia \\ ${ }^{2}$ Institut De Mathematiques et de sciences physiques, Universit D'Abomey Calavi, Benin \\ tesfayeaga2@gmail.com,gdegla@gmail.com,gammeef@gmail.com
}

Received: 28 July 2020; Accepted: 27 January 2021

\begin{abstract}
A uniformly convergent higher-order finite difference scheme is constructed and analyzed for solving singularly perturbed parabolic problems with non-smooth data. This scheme involves an average non-standard finite difference with the Richardson extrapolation method for space variables and second-order finite difference approximation for time direction on uniform meshes. The scheme is shown to be second-order convergent in both temporal and spatial directions. Further, the scheme is proven to be uniformly convergent and also confirmed by numerical experiments. Wide numerical experiments are conducted to support the theoretical results and to demonstrate its accuracy. Concisely, the present scheme is stable, convergent, and more accurate than existing methods in the literature.
\end{abstract}

MSC 2010: 35B25, 35B30, 35B35

Keywords: singularly perturbed parabolic problems, uniformly convergent solution

\section{Introduction}

In this paper, we consider a class of singularly perturbed parabolic problems with non-smooth data whose solutions exhibit strong interior layers due to the discontinuous convection coefficient. These types of problems arise in several branches of engineering and applied mathematics, including convection dominated flows in fluid dynamics, heat, and mass transfer in chemical and nuclear engineering. Singularly perturbed parabolic problems are branded by the occurrence of a small parameter that multiplies the highest order derivative, and there exists a boundary or interior layer where the solutions change rapidly. Solutions of singularly perturbed parabolic problems of convection-diffusion typically contain boundary layers [1-5]. However, interior layers occur if coefficient or source functions or the boundary and/or initial conditions are not sufficiently smooth [6] and [7]. When the perturbation parameter is small, very complex phenomena can 
happen near the point of discontinuity, whose theoretical analysis is not yet well understood. Solving such problems is the most problematic and unresolved problems of fluid mechanics, specifically the behavior of viscous fluids at small viscosity.

Thus, several methods have been established by various authors for different kinds of singularly perturbed parabolic problems with smooth data [8-12]. But, works on problems with non-smooth data are rare. Recently, Chandru et al., [1], proposed a numerical treatment of two-parameter singularly perturbed parabolic convection-diffusion problems with non-smooth data. The optimal error estimate of the upwind scheme on Shishkin-type meshes [10] and an $\varepsilon$-Uniform error estimate of the hybrid numerical scheme [11] for singularly perturbed parabolic problems with interior layers are proposed by Mukherjee and Natesan. These methods are based on piecewise-uniform Shishkin meshes, and most of them are first-order spatial accurate. Therefore, it is necessary to provide a uniformly convergent numerical method with a better accuracy. To achieve this purpose, here we develop a uniformly convergent numerical scheme for solving singularly perturbed parabolic problems with non-smooth data of the form:

$$
\left\{\begin{array}{l}
L_{\varepsilon} u \equiv\left(\varepsilon \frac{\partial^{2} u}{\partial x^{2}}+a(x) \frac{\partial u}{\partial x}-b(x) u-\frac{\partial u}{\partial t}\right)(x, t)=f(x, t),(x, t) \in D \\
u(x, 0)=s(x), \quad x \in \bar{\Omega} \\
u(0, t)=q_{0}(t), \quad u(1, t)=q_{1}(t)
\end{array}\right.
$$

with perturbation parameter $\varepsilon$, satisfies $0<\varepsilon<<1$. The solution domain by $D=\Omega \times(0, T], \quad \Omega=(0,1), \quad \Omega^{-}=(0, d), \quad \Omega^{+}=(d, 1), \quad d \in(0,1), \quad D^{-}=\Omega^{-} \times(0, T]$, $D^{+}=\Omega^{+} \times(0, T], T$ is positive constant. The convection coefficient $a(x)$ and the source term $f(x, t)$ are sufficiently smooth on $D^{-} \cup D^{+}$. Also, the coefficient of the reaction term $b(x)$ is sufficiently smooth on $\bar{D}$ such that

$$
\left\{\begin{array}{l}
b(x) \geq \beta \geq 0, \quad \text { on } \bar{D} \\
|[a]| \leq C,|[f]| \leq C, \quad \text { at } x=d .
\end{array}\right.
$$

and the solution $u(x, t)$ satisfies the following interface conditions

$$
[u]=0, \quad\left[\frac{\partial u}{\partial x}\right]=0, \quad \text { at } x=d .
$$

Let us define the jump of $u$, denoted by $[u]$, across the point of discontinuity $x=d$ by $[u](d, t)=u\left(d^{+}, t\right)-u\left(d^{-}, t\right)$, wherever $u\left(d^{ \pm}, t\right)=\lim _{x \rightarrow d^{ \pm}} u(x, t)$. Due to the presence of discontinuity in the convection coefficient $a(x)$, solution $u(x, t)$ to Eq. (1) possesses interior layers of width $O(\varepsilon)$ in the neighborhood of the point $x=d$. 
The nature of the interior layer depends on the sign of the convection coefficient $a(x)$ on either side of the line of discontinuity [13-18]. Thus, to stress the existence of strong interior layers, consider the following particular condition

$$
\left\{\begin{array}{l}
-\alpha_{1}^{*}<a(x)<-\alpha_{1}<0, \quad x<d \\
\alpha_{2}^{*}>a(x)>\alpha_{2}>0, \quad x>d
\end{array}\right.
$$

Assume that, the coefficient of convection term provided in Eq. (4) is independent of time $t$, and then restrict the discontinuities in the spatial variable $x$ only. Moreover, the data $s(x), q_{0}(t)$ and $q_{1}(t)$ are assumed to be sufficiently smooth on $D$ and satisfy the compatibility conditions at the two corner points $(0,0)$ and $(1,0)$ with $s(0)=q_{0}(0)$ and $s(1)=q_{1}(0)$ and

$$
\left\{\begin{array}{l}
\varepsilon \frac{\partial^{2} s(0)}{\partial x^{2}}+a(0) \frac{\partial s(0)}{\partial x}-b(0) s(0)-f(0,0)=\frac{\partial q_{0}(0)}{\partial t} \\
\varepsilon \frac{\partial^{2} s(1)}{\partial x^{2}}+a(1) \frac{\partial s(1)}{\partial x}-b(1) s(1)-f(1,0)=\frac{\partial q_{1}(0)}{\partial t}
\end{array}\right.
$$

The compatibility conditions at the transition corner point $(d, 0)$ trails similarly, and under these hypotheses the problem in Eqs. (1)-(4) admits a unique solution that one can refer in the book [18].

\section{Formulation of the numerical scheme}

In this section, a numerical scheme will be described by discretizing the time variable on uniform mesh and using the non-standard methodology of Mickens [7, 8] for the space variable. To discretize the time variable with uniform step size $k$, $[0, T]$ is partitioned $0=t_{0}<t_{1}<\ldots<t_{N}=T$ for $t_{n}=n k, k=\frac{T}{N}$. Now, at the point $\left(x, t_{n+\frac{1}{2}}\right)$, the operator in Eq. (1) can be written:

$$
\left(\varepsilon \frac{\partial^{2} u}{\partial x^{2}}+a(x) \frac{\partial u}{\partial x}-b(x) u-\frac{\partial u}{\partial t}\right)\left(x, t_{n+\frac{1}{2}}\right)=f\left(x, t_{n+\frac{1}{2}}\right)
$$

Taylor's series expansion to $u\left(x, t_{n}\right), u\left(x, t_{n+1}\right)$ about the point, $\left(x, t_{n+\frac{1}{2}}\right)$, gives

$$
u_{t}\left(x, t_{n+\frac{1}{2}}\right)=\frac{u\left(x, t_{n+1}\right)-u\left(x, t_{n}\right)}{k}+\tau_{1}
$$


where $\tau_{1}=-\frac{k^{2}}{24} u_{t t t}\left(x, t_{n+0.5}\right)$. This indicates that the error estimate of time discretization is bounded and given by

$$
\left\|E_{n}\right\|_{\infty} \leq C k^{2}
$$

where $C=\frac{1}{24}\left\|u_{t t t}\left(x, t_{n+\frac{1}{2}}\right)\right\|, \forall n=1,2, \ldots N$, is a constant independent of $\varepsilon$ and $k$. Also, let's take the average of all terms of Eq. (6) except for term containing a derivative concerning time, which is written as

$$
\left(\varepsilon \frac{\partial^{2} u}{\partial x^{2}}+a(x) \frac{\partial u}{\partial x}-b(x) u-f\right)\left(x, t_{n+\frac{1}{2}}\right)=\frac{1}{2} L_{x, f}^{*}\left(u\left(x, t_{n+1}\right)+u\left(x, t_{n}\right)\right.
$$

where, $L_{x, f}^{*} u\left(x, t_{n}\right)=L_{x}^{*} u\left(x, t_{n}\right)-f\left(x, t_{n}\right)$,

$$
L_{x}^{*} u\left(x, t_{n}\right)=\left(\varepsilon \frac{\partial^{2} u}{\partial x^{2}}+a(x) \frac{\partial u}{\partial x}-b(x) u\right)\left(x, t_{n}\right) .
$$

Substituting both Eq. (7) and (9) into Eq. (6), we get the problem

$$
\left\{\begin{array}{l}
\left(L_{x}^{*}-\frac{2}{k}\right) u\left(x, t_{n+1}\right)+\left(L_{x}^{*}+\frac{2}{k}\right) u\left(x, t_{n}\right)=f\left(x, t_{n+1}\right)+f\left(x, t_{n}\right) \\
u\left(0, t_{n+1}\right)=q_{0}\left(t_{n+1}\right), \quad u\left(1, t_{n+1}\right)=q_{1}\left(t_{n+1}\right), \quad u(x, 0)=s(x), x \in \bar{\Omega}
\end{array}\right.
$$

This gives the semi-discretize approximation $u\left(x, t_{n+1}\right)$ of Eq. (10) to the exact solution $u(x, t)$ of Eq. (1) at the time levels $t_{n+1}=(n+1) k$. To discretize the space variable, assume that $\bar{\Omega}^{M}$ denotes the partition of $[0,1]$ into $M$ subintervals such that $0=x_{0}<x_{1}<\ldots<x_{\frac{M}{2}}=d<x_{\frac{M}{2}+1} \ldots<x_{M}=1, \quad x_{m}=m h, \quad h=\frac{1}{M}$, and then the tensor-product grids $\bar{D}^{M, N}$. Undertake the notation $U_{m}^{n} \approx u\left(x_{m}, t_{n}\right)$, and the discrete problem to Eq. (10) is given by

$$
\left\{\begin{array}{l}
L_{\varepsilon}^{M, N} U_{m}^{n+1} \equiv\left(L_{M}^{*}-\frac{2}{k}\right) U_{m}^{n+1}+\left(L_{M}^{*}+\frac{2}{k}\right) U_{m}^{n}=f_{m}^{n+1}+f_{m}^{n}, \\
U\left(x_{m}, 0\right)=s\left(x_{m}\right), x_{m} \in \bar{\Omega}^{M} \\
U\left(0, t_{n+1}\right)=q_{0}\left(t_{n+1}\right), u\left(1, t_{n+1}\right)=q_{1}\left(t_{n+1}\right), t_{n+1} \in[0, T]^{N}
\end{array}\right.
$$


where

$$
\left\{\begin{array}{l}
L_{M}^{*} U_{m}^{n+1}=\varepsilon \frac{U_{m+1}^{n+1}-2 U_{m}^{n+1}+U_{m-1}^{n+1}}{\phi_{m}^{2}}+a_{m} \frac{U_{m+1}^{n+1}-U_{m}^{n+1}}{h}-b_{m} U_{m}^{n+1}+\tau_{2} \\
L_{M}^{*} U_{m}^{n}=\varepsilon \frac{U_{m+1}^{n}-2 U_{m}^{n}+U_{m-1}^{n}}{\phi_{m}^{2}}+a_{m} \frac{U_{m+1}^{n}-U_{m}^{n}}{h}-b_{m} U_{m}^{n+1}+\tau_{3}
\end{array}\right.
$$

for the truncation terms in space direction given by

$$
\tau_{2}=-\frac{h}{2}\left(U_{x x}\right)_{m}^{n+1}+O\left(h^{2}\right) \quad \text { and } \quad \tau_{3}=-\frac{h}{2}\left(U_{x x}\right)_{m}^{n}+O\left(h^{2}\right)
$$

Within the nonstandard finite difference methodology of Mickens $[7,8]$, the denominator

$$
\phi_{m}^{2}(\varepsilon, h)=\frac{h \varepsilon}{a_{m}}\left(\exp \left(\frac{h a_{m}}{\varepsilon}\right)-1\right)
$$

Now, incorporating the initial and boundary conditions given, the scheme becomes

$$
\left\{\begin{array}{l}
U_{m}^{0}=s\left(x_{m}\right), \quad \text { for } m=0,1,2, \ldots, M \\
L_{\varepsilon}^{M, N} U_{m}^{n+1}=H_{m}^{n+1}, \quad \text { for } m=1,2, \ldots, M-1 \\
U_{0}^{n+1}=q_{0}\left(t_{n+1}\right), \quad U_{M}^{n+1}=q_{1}\left(t_{n+1}\right), \forall n=0,1, \ldots, N
\end{array}\right.
$$

where

$$
L_{\varepsilon}^{M, N} U_{m}^{n+1}=\overline{A m}_{m-1}^{-} U_{m}^{n+1}+A_{m}^{0} U_{m}^{n+1}+A_{m}^{+} U_{m+1}^{n+1}+\left[\overline{B_{m}^{-}} U_{m-1}^{n}+B_{m}^{0} U_{m}^{n}+B_{m}^{+} U_{m+1}^{n}\right]
$$

and $H_{m}^{n+1}=f_{m}^{n+1}+f_{m}^{n}, m=1,2, \ldots, M-1$ and $n=0,1, \ldots, N$.

Moreover, the coefficients are

$$
\begin{aligned}
& A_{m}^{-}=B_{m}^{-}=\frac{\varepsilon}{\phi_{m}^{2}}, \quad A_{m}^{+}=B_{m}^{+}=\frac{\varepsilon}{\phi_{m}^{2}}+\frac{a_{m}}{h}, \\
& A_{m}^{0}=-\left(\frac{2 \varepsilon}{\phi_{m}^{2}}+\frac{a_{m}}{h}+b_{m}+\frac{2}{k}\right), \text { and } B_{m}^{0}=-\left(\frac{2 \varepsilon}{\phi_{m}^{2}}+\frac{a_{m}}{h}+b_{m}-\frac{2}{k}\right)
\end{aligned}
$$

\section{Consistency and stability of the scheme}

Local truncation errors refer to the differences between the original differential equation and its finite difference approximations at grid points. To investigate 
the consistency of the method, we have the norm of local truncation errors $\left|T_{m}^{n+1}\right|$ obtained in both Eqs. (13) ad (8) for the space and time directions respectively given by

$$
\left|T_{m}^{n+1}\right|=\left|\tau_{2}+\tau_{3}+\tau_{1}\right| \leq C_{1} h+C k^{2}
$$

where the constant are $C_{1}=\left|-\frac{1}{2}\left(U_{x x}\right)_{m}^{n+1}\right|+\left|-\frac{1}{2}\left(U_{x x}\right)_{m}^{n}\right|$ and $C=\frac{1}{24}\left|u_{t t t}\left(x, t_{n+\frac{1}{2}}\right)\right|$.

Thus, the right-hand side hand of Eq. (16) vanishes as $h \rightarrow 0$ and $k \rightarrow 0$ implied that $T_{m}^{n+1} \rightarrow 0$. Hence, the scheme is consistent with the order of convergences $O\left(h+k^{2}\right)$.

The Von Neumann stability technique is applied to investigate the stability of the developed scheme in Eq. (15), by assuming that the solution of Eq. (15) at the grid point $\left(x_{m}, t_{n}\right)$ is given by

$$
U_{m}^{n}=\xi^{n} e^{i \theta m}
$$

where $i=\sqrt{-1}, \theta$ is the real number and $\xi$ denotes the amplitude factor. Then, substituting Eq. (17) into the homogeneous part of Eq. (15) leads to:

$$
\xi=\frac{-\left[B_{m}^{-} e^{-1}+B_{m}^{0}+B_{m}^{+} e^{1}\right]}{A_{m}^{-} e^{-1}+A_{m}^{0}+A_{m}^{+} e^{1}}
$$

Substituting the values of coefficients considered under Eq. (15) into Eq. (18) with stability conditio $|\xi| \leq 1$ is satisfied. Because

$$
|\xi|=\left|-\frac{\frac{\varepsilon}{\phi_{m}^{2}} e^{-1}-\frac{2 \varepsilon}{\phi_{m}^{2}}-\frac{a_{m}}{h}-b_{m}+\frac{2}{k}+\left(\frac{\varepsilon}{\phi_{m}^{2}}+\frac{a_{m}}{h}\right) e^{1}}{\frac{\varepsilon}{\phi_{m}^{2}} e^{-1}-\frac{2 \varepsilon}{\phi_{m}^{2}}-\frac{a_{m}}{h}-b_{m}-\frac{2}{k}+\left(\frac{\varepsilon}{\phi_{m}^{2}}+\frac{a_{m}}{h}\right) e^{1}}\right| \leq 1 .
$$

This leads to:

$$
\begin{aligned}
& \left|\frac{\varepsilon}{\phi_{m}^{2}} e^{-1}-\frac{2 \varepsilon}{\phi_{m}^{2}}-\frac{a_{m}}{h}-b_{m}+\frac{2}{k}+\left(\frac{\varepsilon}{\phi_{m}^{2}}+\frac{a_{m}}{h}\right) e^{1}\right| \leq\left|\frac{\varepsilon}{\phi_{m}^{2}} e^{-1}-\frac{2 \varepsilon}{\phi_{m}^{2}}-\frac{a_{m}}{h}-b_{m}-\frac{2}{k}+\left(\frac{\varepsilon}{\phi_{m}^{2}}+\frac{a_{m}}{h}\right) e^{1}\right|, \\
& \Rightarrow\left|\frac{2}{k}\right| \leq\left|-\frac{2}{k}\right| .
\end{aligned}
$$


Hence, $|\xi| \leq 1$, which implies the developed finite difference scheme in Eq. (15) is unconditionally stable. Therefore, the proposed finite difference scheme is consistent and stable which guarantees it is convergent by Lax's equivalence theorem [19].

\section{Richardson extrapolation}

This technique is a convergence acceleration technique that involves a combination of two computed approximations of a solution. The combination goes out to be an improved approximation $[12,19]$. In this work, we apply the Richardson extrapolation method on the space variable only. So that from Eq. (16), we have

$$
\left|u\left(x_{m}, t_{n+1}\right)-U_{m}^{n+1}\right| \leq C h
$$

where $u\left(x_{m}, t_{n+1}\right)$ and $U_{m}^{n+1}$ are exact and approximate solutions respectively, $C$ is constant free from $\varepsilon$ and $h$. Let $D_{2 M}^{N}$ be the mesh found by dividing each mesh interval in $D_{M}^{N}$ and symbolize the calculation of the solution on $D_{2 M}^{N}$ by $\bar{U}_{m}^{n+1}$. Consider Eq. (19) works for any $h \neq 0$, which implies:

$$
u\left(x_{m}, t_{n+1}\right)-U_{m}^{n+1} \leq C h+R_{M}^{N}, \quad\left(x_{m}, t_{n+1}\right) \in D_{M}^{N} .
$$

Also, consider Eq. (19) works for any $\frac{h}{2} \neq 0$, that leads to:

$$
u\left(x_{m}, t_{n+1}\right)-\bar{U}_{m}^{n+1} \leq C \frac{h}{2}+R_{2 M}^{N}, \quad\left(x_{m}, t_{n+1}\right) \in D_{2 M}^{N} .
$$

where the remainders $R_{M}^{N}$ and $R_{2 M}^{N}$ are $O\left(h^{2}\right)$. A combination of inequalities in Eqs. (20) and (21) leads to $u\left(x_{m}, t_{n+1}\right)-\left(2 \bar{U}_{m}^{n+1}-U_{m}^{n+1}\right) \approx O\left(h^{2}\right)$ which proposes:

$$
\left(U_{m}^{n+1}\right)^{e x t}=2 \bar{U}_{m}^{n+1}-U_{m}^{n+1}
$$

is also an approximate solution of $u\left(x_{m}, t_{n+1}\right)$. The solution obtained by Eq. (22) approximates the solution with an estimated truncation error

$$
\left|u\left(x_{m}, t_{n+1}\right)-\left(U_{m}^{n+1}\right)^{e x t}\right| \leq C h^{2} .
$$

Thus, from Eq. (23) for the order in the spatial direction with Eqs. (7) and (8) for the order of temporal direction, we can conclude that for $\left(U_{m}^{n+1}\right)^{\text {ext }}$ be the solution of Eq. (15) and $u_{m}^{n+1}$ be the solution to Eq. (1) at the grid point $\left(x_{m}, t_{n+1}\right)$ : 


$$
\max _{0 \leq m \leq M}\left|u_{m}^{n+1}-\left(U_{m}^{n+1}\right)^{e x t}\right| \leq C\left(h^{2}+k^{2}\right), n=0,1, \ldots, N,
$$

where a constant $C$ is independent of $\varepsilon, h$, and $k$.

\section{Numerical illustrations}

To demonstrate the applicability of the developed scheme computationally, we consider three examples. The exact solution for such types of problems is not available, so that maximum absolute errors at all the mesh points are evaluated before and after the Richardson extrapolation using the formula

$$
E_{\varepsilon}^{M, N}=\max _{0 \leq m \leq M ; 0 \leq n \leq N}\left|U_{m}^{n+1}-U_{2 m}^{n+1}\right| \text { and } E R_{\varepsilon}^{M, N}=\max _{0 \leq m \leq M ; 0 \leq n \leq N}\left|\left(U_{m}^{n+1}\right)^{e x t}-\left(U_{2 m}^{n+1}\right)^{e x t}\right|
$$

respectively, where $U_{m}^{n+1}$ is an approximate solution obtained using a constant space mash size $h$ and time step $k$, and $U_{2 m}^{n+1}$ is also an approximate solution produced using space step size $\frac{h}{2}$.

Example 1. Consider the singularly perturbed parabolic problem

$$
\begin{aligned}
& \left\{\begin{array}{l}
\varepsilon u_{x x}+a(x) u_{x}-x(1-x) u-u_{t}=f(x, t),(x, t) \in(0,1) \times(0,1] \\
u(x, 0)=0, \quad 0 \leq x \leq 1 \\
u(0, t)=t^{2}, \quad u(1, t)=0, \quad 0<t \leq 1
\end{array}\right. \\
& a(x)=\left\{\begin{array}{ll}
-(1+x(1+x)), \quad 0 \leq x \leq 0.5 \\
(1+x(1+x)), & 0.5<x \leq 1
\end{array} \text { and } f(x, t)= \begin{cases}2\left(1+x^{2}\right) t^{2}, & 0 \leq x \leq 0.5 \\
3\left(1+x^{2}\right) t^{2}, & 0.5<x \leq 1\end{cases} \right.
\end{aligned}
$$

A comparison of maximum absolute errors for Example 1 is given in Table 1 with solution behavior in Figure 1a.

Example 2. Consider the parabolic problem

$$
\begin{aligned}
&\left\{\begin{array}{l}
\varepsilon u_{x x}+a(x) u_{x}-u_{t}=f(x, t),(x, t) \in(0,1) \times(0,1] \\
u(x, 0)=0, \quad 0 \leq x \leq 1 \\
u(0, t)=0=u(1, t), \quad 0<t \leq 1
\end{array}\right. \\
& a(x)=\left\{\begin{array}{l}
-\left(2+x^{2}\right), \quad 0 \leq x \leq 0.5 \\
3-x^{2}, \quad 0.5<x \leq 1
\end{array} \text { and } f(x, t)=\left\{\begin{array}{l}
2 x \exp (-t) t^{2}, \quad 0 \leq x \leq 0.5 \\
2(1-x) \exp (-t) t^{2}, \quad 0.5<x \leq 1
\end{array}\right.\right.
\end{aligned}
$$

The computed maximum absolute errors and the corresponding order of convergence for Example 2 are presented in Tables 2 and 3 in the cases of before and after Richardson extrapolation. Also, the solution behavior in Figure $1 \mathrm{~b}$ and $\log -\log$ plot in Figure 2a are given. 
Example 3. Consider the problem

$$
\begin{gathered}
\left\{\begin{array}{l}
\varepsilon u_{x x}+a(x) u_{x}-u_{t}=f(x, t),(x, t) \in(0,1) \times(0,1] \\
u(x, 0)=0, \quad 0 \leq x \leq 1 \\
u(0, t)=t^{2}, \quad u(1, t)=0, \quad 0<t \leq 1
\end{array}\right. \\
\text { where } a(x)=\left\{\begin{array}{ll}
-1, & 0 \leq x \leq 0.5 \\
1, & 0.5<x \leq 1
\end{array} \text { and } f(x, t)=\left\{\begin{array}{l}
2 x t, \quad 0 \leq x \leq 0.5 \\
2(1-x) t, \quad 0.5<x \leq 1
\end{array}\right.\right.
\end{gathered}
$$

A comparison of maximum absolute errors for Example 3 is given in Table 4 with a log-log plot in Figure 2b.

\begin{tabular}{|c|c|c|c|c|}
\hline$\varepsilon \downarrow M=N \rightarrow$ & 32 & 64 & 128 & 256 \\
\hline \multicolumn{5}{|l|}{ Present Method } \\
\hline $10^{-1}$ & $1.3124 \mathrm{e}-03$ & $2.1065 \mathrm{e}-04$ & $5.3718 \mathrm{e}-05$ & $1.3570 \mathrm{e}-05$ \\
\hline $10^{-4}$ & $8.1406 \mathrm{e}-04$ & $3.3685 \mathrm{e}-04$ & $1.4815 \mathrm{e}-04$ & $6.8379 \mathrm{e}-05$ \\
\hline $10^{-6}$ & $8.1406 \mathrm{e}-04$ & $3.3685 \mathrm{e}-04$ & $1.4815 \mathrm{e}-04$ & $6.8379 \mathrm{e}-05$ \\
\hline $10^{-8}$ & $8.1406 \mathrm{e}-04$ & $3.3685 \mathrm{e}-04$ & $1.4815 \mathrm{e}-04$ & $6.8379 \mathrm{e}-05$ \\
\hline $10^{-10}$ & $8.1406 \mathrm{e}-04$ & $3.3685 \mathrm{e}-04$ & $1.4815 \mathrm{e}-04$ & $6.8379 \mathrm{e}-05$ \\
\hline \multicolumn{5}{|l|}{ Results in [15] } \\
\hline $10^{-1}$ & $4.5469 \mathrm{e}-03$ & $2.1384 \mathrm{e}-03$ & $1.0448 \mathrm{e}-03$ & $5.1766 \mathrm{e}-04$ \\
\hline $10^{-4}$ & $7.3215 \mathrm{e}-02$ & $2.7267 \mathrm{e}-02$ & $8.3339 \mathrm{e}-03$ & $2.7829 \mathrm{e}-03$ \\
\hline $10^{-6}$ & $7.3246 \mathrm{e}-02$ & $2.7273 \mathrm{e}-02$ & $8.3349 \mathrm{e}-03$ & $2.7828 \mathrm{e}-03$ \\
\hline $10^{-8}$ & $7.3246 \mathrm{e}-02$ & $2.7273 e-02$ & $8.3349 \mathrm{e}-03$ & $2.7828 \mathrm{e}-03$ \\
\hline $10^{-10}$ & $7.3246 \mathrm{e}-02$ & $2.7273 e-02$ & $8.3349 \mathrm{e}-03$ & $2.7827 \mathrm{e}-03$ \\
\hline
\end{tabular}

Table 1. Comparison of maximum absolute errors for Example 1

\begin{tabular}{|c|c|c|c|c|c|}
\hline$\varepsilon \downarrow N$ & 32 & 64 & 128 & 256 & 512 \\
\hline \multicolumn{6}{|c|}{ After Extrapolation } \\
\hline $10^{-1}$ & $2.3784 \mathrm{e}-04$ & $6.8070 \mathrm{e}-05$ & $1.7754 \mathrm{e}-05$ & $4.5055 \mathrm{e}-06$ & $1.1331 \mathrm{e}-06$ \\
\hline $10^{-4}$ & $1.3531 \mathrm{e}-05$ & $3.4015 \mathrm{e}-06$ & $8.5267 \mathrm{e}-07$ & $2.1345 \mathrm{e}-07$ & $5.5331 \mathrm{e}-08$ \\
\hline $10^{-6}$ & $1.3531 \mathrm{e}-05$ & $3.4015 \mathrm{e}-06$ & $8.5267 \mathrm{e}-07$ & $2.1345 \mathrm{e}-07$ & $5.5331 \mathrm{e}-08$ \\
\hline $10^{-8}$ & $1.3531 \mathrm{e}-05$ & $3.4015 \mathrm{e}-06$ & $8.5267 \mathrm{e}-07$ & $2.1345 \mathrm{e}-07$ & $5.5331 \mathrm{e}-08$ \\
\hline $10^{-10}$ & $1.3531 \mathrm{e}-05$ & $3.4015 \mathrm{e}-06$ & $8.5267 \mathrm{e}-07$ & $2.1345 \mathrm{e}-07$ & $5.5331 \mathrm{e}-08$ \\
\hline \multicolumn{6}{|c|}{ Before Extrapolation } \\
\hline $10^{-1}$ & $1.0164 \mathrm{e}-03$ & $3.8963 \mathrm{e}-04$ & $1.6087 \mathrm{e}-04$ & $7.1577 \mathrm{e}-05$ & $3.3541 \mathrm{e}-05$ \\
\hline $10^{-4}$ & $9.0542 \mathrm{e}-04$ & $4.5979 \mathrm{e}-04$ & $2.3167 \mathrm{e}-04$ & $1.1628 \mathrm{e}-04$ & $5.8250 \mathrm{e}-05$ \\
\hline $10^{-6}$ & $9.0542 \mathrm{e}-04$ & $4.5979 \mathrm{e}-04$ & $2.3167 \mathrm{e}-04$ & $1.1628 \mathrm{e}-04$ & $5.8250 \mathrm{e}-05$ \\
\hline $10^{-8}$ & $9.0542 \mathrm{e}-04$ & $4.5979 \mathrm{e}-04$ & $2.3167 \mathrm{e}-04$ & $1.1628 \mathrm{e}-04$ & $5.8250 \mathrm{e}-05$ \\
\hline $10^{-10}$ & $9.0542 \mathrm{e}-04$ & $4.5979 \mathrm{e}-04$ & $2.3167 \mathrm{e}-04$ & $1.1628 \mathrm{e}-04$ & $5.8250 \mathrm{e}-05$ \\
\hline
\end{tabular}

Table 2. Maximum absolute errors for Example 2 when $M=N$ 
Table 3. Rate of convergence for Example 2, when $M=N$

\begin{tabular}{|c|c|c|c|c|}
\hline$\varepsilon \downarrow N$ & 32 & 64 & 128 & 256 \\
\hline \multicolumn{5}{|c|}{ After Extrapolation } \\
\hline $10^{-1}$ & 1.8049 & 1.9389 & 1.9784 & 1.9914 \\
\hline $10^{-4}$ & 1.9920 & 1.9961 & 1.9981 & 1.9477 \\
\hline $10^{-6}$ & 1.9920 & 1.9961 & 1.9981 & 1.9477 \\
\hline $10^{-8}$ & 1.9920 & 1.9961 & 1.9981 & 1.9477 \\
\hline $10^{-10}$ & 1.9920 & 1.9961 & 1.9981 & 1.9477 \\
\hline \multicolumn{5}{|c|}{ Before Extrapolation } \\
\hline $10^{-1}$ & 1.3833 & 1.2762 & 1.1683 & 1.0936 \\
\hline $10^{-4}$ & 0.9776 & 0.9889 & 0.9945 & 0.9973 \\
\hline $10^{-6}$ & 0.9776 & 0.9889 & 0.9945 & 0.9973 \\
\hline $10^{-8}$ & 0.9776 & 0.9889 & 0.9945 & 0.9973 \\
\hline $10^{-10}$ & 0.9776 & 0.9889 & 0.9945 & 0.9973 \\
\hline
\end{tabular}

Table 4. Comparison of maximum absolute errors for Example 3 when $M=N$

\begin{tabular}{|c|c|c|c|c|c|}
\hline$\varepsilon \downarrow N$ & 32 & 64 & 128 & 256 & 512 \\
\hline \multicolumn{6}{|c|}{ After Extrapolation } \\
\hline $10^{-1}$ & $5.3343 \mathrm{e}-04$ & $1.3647 \mathrm{e}-04$ & $3.4315 \mathrm{e}-05$ & $8.5912 \mathrm{e}-06$ & $2.1486 \mathrm{e}-06$ \\
\hline $10^{-5}$ & $9.4849 \mathrm{e}-05$ & $2.9819 \mathrm{e}-05$ & $1.0681 \mathrm{e}-05$ & $3.8488 \mathrm{e}-06$ & $1.3861 \mathrm{e}-06$ \\
\hline $10^{-10}$ & $9.4849 \mathrm{e}-05$ & $2.9819 \mathrm{e}-05$ & $1.0681 \mathrm{e}-05$ & $3.8488 \mathrm{e}-06$ & $1.3861 \mathrm{e}-06$ \\
\hline \multicolumn{6}{|c|}{ Before Extrapolation } \\
\hline $10^{-1}$ & $1.0686 \mathrm{e}-03$ & $2.7073 \mathrm{e}-04$ & $6.7910 \mathrm{e}-05$ & $1.6992 \mathrm{e}-05$ & $4.2488 \mathrm{e}-06$ \\
\hline $10^{-5}$ & $2.7667 \mathrm{e}-03$ & $1.4248 \mathrm{e}-03$ & $7.2263 \mathrm{e}-04$ & $3.6385 \mathrm{e}-04$ & $1.8256 \mathrm{e}-04$ \\
\hline $10^{-10}$ & $2.7667 \mathrm{e}-03$ & $1.4248 \mathrm{e}-03$ & $7.2263 \mathrm{e}-04$ & $3.6385 \mathrm{e}-04$ & $1.8256 \mathrm{e}-04$ \\
\hline \multicolumn{6}{|c|}{ Results in [14] } \\
\hline $10^{-1}$ & $4.6571 \mathrm{e}-3$ & $1.9099 \mathrm{e}-3$ & $8.8214 \mathrm{e}-4$ & $4.2631 \mathrm{e}-4$ & $2.0976 \mathrm{e}-4$ \\
\hline $10^{-5}$ & $9.9736 \mathrm{e}-3$ & $4.1094 \mathrm{e}-3$ & $1.7641 \mathrm{e}-3$ & $8.2587 \mathrm{e}-4$ & $3.9692 \mathrm{e}-4$ \\
\hline $10^{-10}$ & $9.9737 \mathrm{e}-3$ & $4.1095 \mathrm{e}-3$ & $1.7641 \mathrm{e}-3$ & $8.2580 \mathrm{e}-4$ & $3.9691 \mathrm{e}-4$ \\
\hline
\end{tabular}

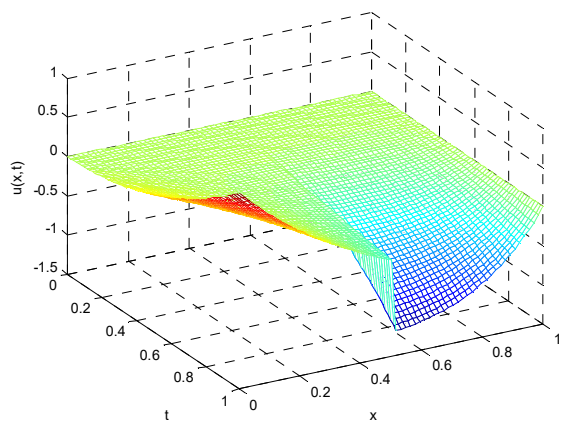

(a) Example 1

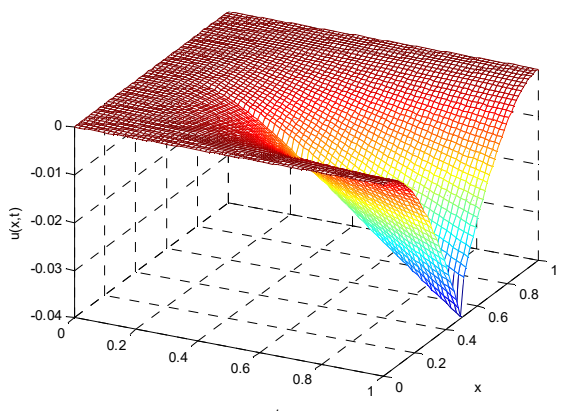

(b) Example 2

Fig. 1. Numerical solution when $\varepsilon=10^{-4}, M=N=64$ for Examples 1 and 2 


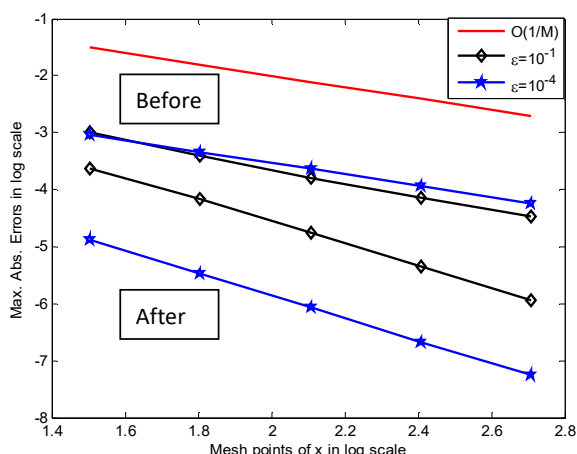

(a) Example 1

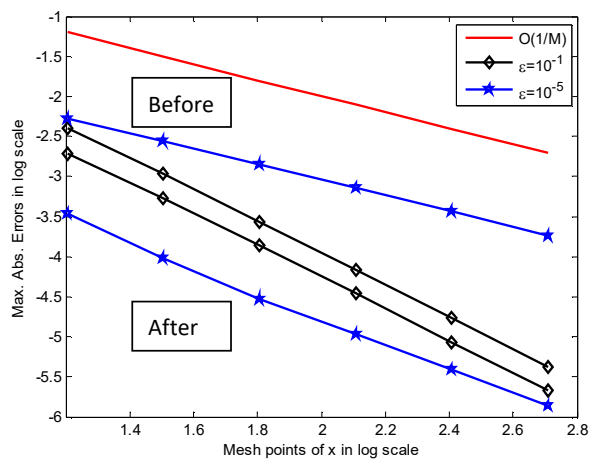

(b) Example 3

Fig. 2. Log-log plot of maximum absolute errors before and after extrapolation at the number of intervals for Examples 2 and 3

\section{Conclusion}

In this paper, we have developed and analyzed a uniformly convergent higher-order finite difference scheme for solving singularly perturbed parabolic problems with non-smooth data. To develop this scheme, we use a uniform mesh for the temporal direction and followed by a nonstandard finite difference methodology of Mickens in the spatial direction. We have recognized the maximum principle and stability results for continuous and discrete problems, and their decomposition in smooth and layer components. It has been shown theoretically that the developed scheme is uniformly convergent second-order accuracy and confirmed with experimental results in Table 3. Also, the accuracy of computational verification tested and compared as displayed in Tables 1,2, and 4 for three considered model examples. Moreover, we also observed that the proposed scheme has a better numerical accuracy compared to the existing methods in the literature. To verify the interior layer that happens due to a discontinuous convection coefficient and source function. This layer behavior is simulated in Figure 1. From Figure 2, we illustrate the contribution of the Richardson extrapolation technique gives a more accurate solution with a high rate of convergence.

Generally, the proposed method is uniformly convergent of order two (higher-order) for solving singularly perturbed parabolic problems with non-smooth data. Furthermore, the method is stable, convergent, and gives a more accurate solution than the existing methods in the literature.

\section{References}

[1] Chandru, M., Das, P., \& Ramos, H. (2018). Numerical treatment of two-parameter singularly perturbed parabolic convection-diffusion problems with non-smooth data. Mathematical Method in Applied Sciences, 1-29, DOI: 10.1002/mma.5067.

[2] Das, P., \& Mehrman, V. (2015). Numerical solution of singularly perturbed convection-diffusionreaction problems with two small parameters. BIT Numerical Mathematics, DOI: 10.1007/s10543015-0559-8 
[3] Gowrisankar, S., \& Srinivasan, N. (2014). Robust numerical scheme for singularly perturbed convection-diffusion parabolic initial-boundary-value problems on equidistributed grids. Computer Physics Communications, 185, 2008-2019.

[4] Gracia, J.L., \& O'Riordan, E. (2019). Singularly perturbed reaction-diffusion problems with discontinuities in the initial and/or the boundary data. Journal of Computational and Applied Mathematics, 370, 112638, DOI: 10.1016/j.cam.2019.112638.

[5] Gupta, V., Kadalbajoo, M.K., \& Dubey, R.K. (2018). A parameter-uniform higher-order finite difference scheme for singularly perturbed time-dependent parabolic problem with two small parameters. International Journal of Computer Mathematics, DOI: 10.1080/00207160.2018. 1432856.

[6] Jha, A., \& Kadalbajoo, M.K. (2015). A robust layer adapted difference method for singularly perturbed two-parameter parabolic problems. International Journal of Computer Mathematics, 92, 1204-1221.

[7] Mickens, R.E. (2005). Advances in the Applications of Nonstandard Finite Difference Schemes. Singapore: World Scientific Publishing.

[8] Mickens, R.E. (1994). Nonstandard Finite Difference Models of Differential Equations. Singapore: World Scientific Publishing.

[9] Miller, H.J.J., O’Riordan, E., \& Shishkin, I.G. (1996). Fitted Numerical Methods for Singular Perturbation Problems, Error Estimate in the Maximum Norm for Linear Problems in One and Two Dimensions. Singapore: World Scientific Publishing.

[10] Mukherjee, K., \& Natesan, S. (2011). Optimal error estimate of the upwind scheme on Shishkintype meshes for singularly perturbed parabolic problems with discontinuous convection coefficients. BIT Numerical Mathematics, 51, 289-315, DOI: 10.1007/s10543-010-0292-2.

[11] Mukherjee, K., \& Natesan, S. (2011). $\varepsilon$ - Uniform error estimate of a hybrid numerical scheme for singularly perturbed parabolic problems with interior layers. Numerical Algorithms, 58, 103-141, DOI: 10.1007/s11075-011-9449-6.

[12] Tesfaye, A.B., Gemechis, F.D., \& Degla, G.A. (2020). Robust finite difference method for singularly perturbed two-parameter parabolic convection-diffusion problems. International Journal of Computational Methods, DOI: 10.1142/S0219876220500346.

[13] Munyakazi, J.B. (2015). A robust finite difference method for two-parameter parabolic convection-diffusion problems. An International Journal of Applied Mathematics and Information Sciences, 9, 2877-2883.

[14] Munyakazi, J.B., \& Patidar, K.C. (2013). A fitted numerical method for singularly perturbed parabolic reaction-diffusion problems. Computational and Applied Mathematics, 32, 509-519.

[15] Munyakazi, J.B., Patidar, K.C., \& Mbani, T.S. (2019). A fitted numerical method for parabolic turning point singularly perturbed problems with an interior layer. Numerical Methods for Partial Differential Equations, 35, 2407-2422.

[16] O'Riordan, E., \& Shishkin, G.I. (2004). Singularly perturbed parabolic problems with non-smooth data. Journal of Computational and Applied Mathematics, 166, 233-245.

[17] Rajan, M.P., \& Reddy, G.D. (2015). An iterative technique for solving singularly perturbed parabolic PDE. Journal of Applied Mathematics and Computing, DOI: 10.1007/s12190-0150866-x.

[18] Roos, G.H, Stynes, M., \& Tobiska, L. (2008). Robust Numerical Methods for Singularly Perturbed Differential Equations, Convection-Diffusion-Reaction and Flow Problems, Second Edition. Berlin, Heidelberg: Springer-Verlag.

[19] Tesfaye, A.B., Gemechis, F.D., \& Degla, G.A. (2019). Higher-order fitted operator finite difference method for two-parameter parabolic convection-diffusion problems. International Journal of Engineering and Applied Sciences (IJEAS), 11, 455-467. 